\title{
Review
}

Mandile Samantha Thobela*, Mantombi Rebecca Maseme and Bonginkosi Mthandeni Duma

\section{An overview of the National Biobank of the National Health Laboratory Service: a South African national treasure for biological resources}

https://doi.org/10.1515/labmed-2021-0101

Received August 12, 2021; accepted February 14, 2022;

published online March 4, 2022

\begin{abstract}
The National Biobank of the National Health Laboratory Service (NHLS) is a national treasure established to serve as support infrastructure for the provision of high quality human biological materials for research purposes and it represents the first of its kind in South Africa. This article aims to demonstrate the alignment of the NHLS Biobank to international best practices and guidelines with reference to the 13 sections of the International Society of Biological and Environmental Repositories (ISBER) Best Practices for Repositories (4th ed.). The NHLS Biobank has implemented procedures and management strategies that are technical best practices covering the lifecycle of biobanking (collection, processing, storage and dissemination of human biological materials) while having respect for ethical and regulatory processes, upholding the interest of the donors. ISBER best practices are invaluable sources of guidance and benchmarking on the guiding principles has enabled the NHLS Biobank to develop into an entity with infrastructure and operational activities that support its short-term and long-term objectives that are set out in the business plan.
\end{abstract}

Keywords: human biological materials; international best practices; National Biobank; quality management; research; South Africa.

\footnotetext{
*Corresponding author: Mandile Samantha Thobela, National Health Laboratory Service, National Biobank, 25 Hospital Street, Constitution Hill, Johannesburg 2000, South Africa, Phone: +27 11 7126432, Fax: +27 11 7126530, E-mail: MandileT@nioh.ac.za. https://orcid.org/ 0000-0002-9140-4523

Mantombi Rebecca Maseme, National Health Laboratory Service, National Biobank, Johannesburg, South Africa

Bonginkosi Mthandeni Duma, National Health Laboratory Service, National Biobank, Johannesburg, South Africa; and National Institute for Occupational Diseases, Quality Assurance, Johannesburg, South Africa
}

\section{Background}

Retrospective to the late 1990s when the term 'biobank' first appeared in literature, it has since been defined by many authors across different fields [1-7]. The organised process of collecting, processing, storage and dissemination of biological samples and associated data primarily for research purposes are some of the common constituents that frequently appear when a biobank is defined [2, 3, 5-7]. Biobanks are established as support infrastructure for diagnostic, therapeutic or research purposes and these biobanks can either be population-based, disease-oriented, hospital-or academic-based, networked, or run by the government, non-profit organizations, or commercial companies among others [2, 5, 7-10].

The concept of biobanking is becoming a common feature of relevance in biomedical research consequent to the potential to aid in innovation and new developments in diagnosis, therapies and human disease research $[2,3$, $6,7,9-11]$. The evolution of biobanks, particularly for human biobanks, is accompanied by complexities that are consequent to their multi-disciplinary nature involving science, genetics, society, ethics, legislation and politics [5-7, 9-13].

Heterogeneity and a lack of standardization attributed to normative and legal aspects for majority of the established biobanks is currently what affects sharing of samples and data for research between biobanks [14-17]. A sound quality management system (QMS) is imperative in ensuring that services and products provided by a biobank are fit for purpose [18]. Organisational networks and societies for biobanks such as the International Society of Biological and Environmental Repositories (ISBER) have been instrumental in spreading excellence in biobanking and cohort building [4, 7, 10, 19-22]. These networks and societies provide assistance in all aspects of biobanking for member biobanks to achieve best practices at the international level of biobanking $[19,20]$. Prior to the development and official release of the ISO 20378:2018 biobank standard, there was no relevant international 
standard in existence that could be applied to all activities of a biobank [3, 10, 23]. The establishment of many biobanks relied on international best practices and guidelines intended for biobanks as well as a variety of international quality standards for efficiency in operation of activities such as ISO 9001and ISO 17025 standards [7, 10, 23-25]. ISO 9001 was examined and found to be applicable for the implementation of QMSs, client satisfaction and continuous improvement for all types of biobanks [10, 23].

While ISO 9001 is concerned with the overall management, ISO 17025 demonstrates competence of the accredited biobank to provide quality samples and data that are accurate and reliable [23, 26]. ISO 20387:2018 which is concerned with the general requirements for biobanking was officially published as a guidance document to enable biobanks to operate within an internationally accepted standardized framework [7, 23, 27]. The requirements of this standard promotes quality, trust, and reliability of samples and their data in order to yield valuable research results [7, 23, 27]. Harmonization of biobanks which is reliant on an internationally accepted quality standard will enable cooperation between biobanks and stakeholders and subsequently foster inter-country and-intra-country sharing of biological samples and their data to advance innovation and new developments in biomedical research $[8,10]$.

The perception of biobanks being a feasible model for developed countries as opposed to resource-poor settings is now a thing of the past as the establishment of biobanks rapidly spills over to developing countries such as countries in the African continent $[28,29]$. South Africa (SA) is amongst these developing countries, which is home to the National Health Laboratory Service (NHLS) National Biobank. To date, the NHLS Biobank represents the first and only established population-based national repository in SA which has been in operation since the year 2012. In SA, there are biobanks concerned with the collection and management of biological specimens of human, animal, and plant origin across different organisation [30]. According to literature, known human-based biobanks in South are either private, university institutions, publicprivate partnerships, national and data registry biobanks that are concerned with various sample source and are located in various parts of SA [30].

This article aims to provide an overview of how the NHLS Biobank, being the only one of its kind in SA, is aligned and acquiescent to international best practices and guidelines for biobanks with special reference to the 13 sections of ISBER Best Practices for Repositories (4th ed.) [20]. ISBER Best Practices for Repositories is not a legal constitution or regulation for repositories, but merely guiding principles collated from the experience of its members and input from repository professionals for the successful implementation and operation of repositories and subscribing to guidance contained herein is exclusively on a voluntary basis [20].

\section{Section A: Repository planning considerations}

The NHLS Biobank which is situated at the National Institute for Occupational Health (NIOH), a division of the NHLS, is a physical repository of national importance to SA and global researchers [31]. It was established in 2012 as a not-for-profit entity purposefully designed to serve as a research infrastructure to support the research community undertaking research to alleviate the growing burden of communicable and non-communicable diseases in SA [31]. The NHLS Biobank is a population-based human biobank with a capacity to store a wide variety of samples. The NHLS Biobank scope of practice is to formally manage, in an organised manner, the collection, processing, storage, retrieval and distribution of human biological materials (HBMs) and their associated data for research purposes both now and in future nationally [31, 32]. The NHLS Biobank infrastructure has the capacity to store 4 million samples; $39 \%$ of its storage capacity is currently utilized with a total of 1,558144 samples (buffy coat $n=6,752$; cytology microscope slides $n=200,000$; cytology block samples $n=6,709$; DNA $n=3,466$; histology microscope slides $n=766,500$; histology block samples $n=75,750$; Microbanks $n=189$; plasma $n=351,017$; serum $n=146,360$; and urine $n=1,401$ ) in storage as illustrated in Figure 1.

Sample collection is handled by the clients who determine their specific research interests (needs), donors and donor characteristics that they require. All samples types were collected from participants who were patients presenting with HIV, TB, metabolic diseases (e.g. diabetes) as well as patients requiring cytopathology and histopathology tests at government hospital settings. Table 1 is a representation of the sample profiles and downstream applications for samples currently in storage. Notably, due to the long-term nature of biobank research, the specific research details and associated downstream application(s) is not always possible.

The NHLS Biobank acknowledges the genetic diversity in SA and recognises it as a national treasure [9]. Availability and accessibility of HBMs within legal and ethical boundaries will foster national research initiatives 


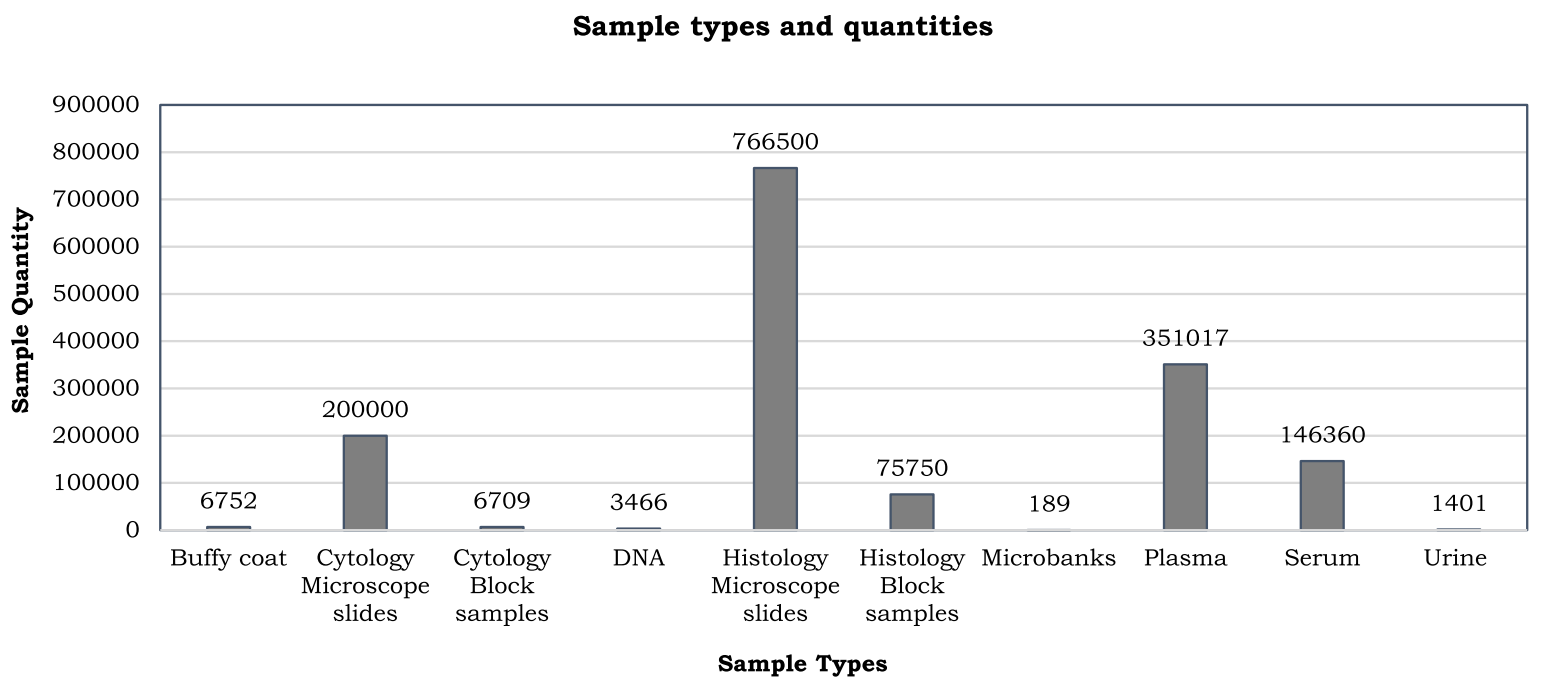

Figure 1: Sample types and quantities in storage at the NHLS Biobank.

Table 1: Sample profiles and downstream application(s).

\begin{tabular}{ll}
\hline Sample type & Downstream application(s) \\
\hline Buffy coat & Qualitative and nucleic acid testing; e.g. HIV visit samples \\
Cytology and histology microscope slides and & $\begin{array}{l}\text { Pathology referrals, research purposes, teaching and training of medical students and } \\
\text { medical professionals }\end{array}$ \\
block samples & Genetic testing, e.g. for TB \\
DNA & Bacterial cell culture and viability testing \\
Microbanks & Qualitative and nucleic acid testing, e.g. HIV positive samples from patients on ART \\
Serum & Non-communicable disease testing (NCDs), e.g. diabetes \\
Urine & Qualitative and nucleic acid testing, e.g. HIV positive samples from patients on ART \\
Plasma &
\end{tabular}

and capacity building. Consequently, sample and data protection and control while safeguarding the interests of research participants.

The successful operation and management of the NHLS Biobank activities is owed to the governance mechanisms that support transparency, accountability and oversight. The NHLS Board, CEO and the NIOH executive are in charge of the NHLS Biobank. The NHLS gets monthly updates of the biobank from the Projects Manager of the NHLS Biobank. The Projects Manager reports to the $\mathrm{NIOH}$ Executive and the operation of the biobank are reported on a monthly basis to the Executive Committee (EXCO) of the NHLS, who then report to the NHLS board. The NHLS board reports to the Minister of Health. An annual report is produced to inform public and stakeholders of the biobank operations.

The NHLS Biobank has an elaborate business plan and governance framework that covers the entire biobanking lifecycle, this is documented in quality manuals, policies and standard operating procedures (SOPs) to ensure correct implementation of crucial components of biobanking and these documents are made accessible to stakeholders (Table 2) [32, 33].

Legitimacy of biobanks and subsequently its social license is heavily reliant on the governance structure [34]. The NHLS Biobank upholds governance by adopting the commonly used governance strategies in biobanking. These strategies include communication, compliance, expert advice, external review, internal procedures and partnerships [34].

\section{Communication}

The NHLS Biobank employs various communication strategies to create awareness of the existence of the national treasure and its structure and activities. To expand its reach globally, the NHLS Biobank has Internet presence where information about the biobank is provided in the form of a video and written content. Furthermore, communication about the biobank is distributed through presentations as a primary channel to engage health professionals, researchers, scientific communities and the general public. 
Table 2: Regulatory framework and compliance instruments.

\begin{tabular}{|c|c|}
\hline Acts of parliament and guidelines & Compliance aspect \\
\hline Constitution Act 108 of 1996 & Informed consent (IC) \& privacy sections: $12(2)(c)-I C ; 14(d)$-privacy \\
\hline $\begin{array}{l}\text { Health professions Act } 56 \text { of } 1974 \text { \& HPCSA codes of practice for ethical } \\
\text { conduct }\end{array}$ & $\begin{array}{l}\text { Regulation of biobank medical scientists to ensure competence } \\
\text { and sound ethical conduct }\end{array}$ \\
\hline National health Act 61 of 2003 (NHA) & $\begin{array}{l}\text { IC \& confidentiality sections: } 71(b) \&(c)-I C ; 14 \text {-confidentiality; } \\
\text { 73-HREC approval for research }\end{array}$ \\
\hline NDoH ethics in health research principles, processes and structures 2015 & IC, confidentiality, privacy, HREC approval \\
\hline NHA material transfer agreement (NMTA) & Material transfer \\
\hline Occupational health and safety Act 85 of 1993 & Employee safety \\
\hline Protection of personal information Act 4 of 2013 & Protection of donor personal information \\
\hline QMS instruments & Compliance aspect \\
\hline \multicolumn{2}{|l|}{ Standard } \\
\hline SANS 9001:2015 SA national standard & $\begin{array}{l}\text { Scope: Biobank (sample storage) \& receiving (NIO departments)-All } \\
\text { clauses }\end{array}$ \\
\hline \multicolumn{2}{|l|}{ SOPs } \\
\hline DNA extraction from frozen tissue & Sample preparation \\
\hline Temperature monitoring software for biobank freezers and fridges & Sample integrity during storage \\
\hline Nanophotometer operation, use and maintenance & Sample preparation \\
\hline $\begin{array}{l}\text { Sample transporting, collection, receiving and storage for biobanking } \\
\text { purposes }\end{array}$ & Pre-processing, processing, storage (sample and data) \\
\hline Enrolling of new users on biometric access software & Restricted access for data and sample protection \\
\hline Disposing of unused biobank samples & Preventing inappropriate use to respect donor autonomy (IC) \\
\hline Biobank quality manual & $\begin{array}{l}\text { QMS for all biobank and sample receiving ( } \mathrm{NIOH} \text { departments) } \\
\text { processes }\end{array}$ \\
\hline $\begin{array}{l}\text { Operation and maintenance of } \mathrm{O}_{2} \text { depletion monitors, fume extraction } \\
\text { hood }\end{array}$ & Employee safety \\
\hline $\begin{array}{l}\text { Operation, use and maintenance of storage equipment: Freezers, fridges, } \\
\mathrm{LN}_{2} \text { tanks }\end{array}$ & Sample integrity during storage \\
\hline $\begin{array}{l}\text { Ethics procedure, MTA, obtaining IC, withdrawal of IC, governance } \\
\text { procedure }\end{array}$ & Ethics compliance in line with the laws and guidelines above \\
\hline Automated (software) and manual (Excel) sample registration & Organized sample storage \\
\hline Disaster management plan & Sample and data protection \\
\hline International air transport association (IATA) certification & Compliance aspect \\
\hline Sample shipping & Shipping of class 6.2 dangerous goods \\
\hline
\end{tabular}

Posters and brochures of the biobank are also made available in NHLS facilities, and guided tours of the biobank are also conducted for interested parties, existing clients and stakeholders.

\section{Compliance}

Various national and international policies and guidelines for regulating health research are adopted to guide the NHLS Biobank activities and research. The regulatory framework and compliance instruments are outlined in Table 2. There is currently a lack of a holistic regulatory framework for research biobanks in SA and the African continent as a whole [35-37]. The legal and ethical frameworks in existence are subjective and lack coherence [35-37]. Subjectivity results in confusion as to what is permissible for research biobanks involved in sample and data collection, storage, and sharing. Existing legal and ethical frameworks in Africa needs to be streamlined to support health research and protect the interests of sample donors without ambiguity [35-37].

\section{Expert advice}

Oversight of the biobank is achieved through a steering committee consisting of experts in research, legal and ethical codes, and scientific management. The constituents of the steering committee are concerned with the regulatory, statutory, ethical, and best practice requirements within the biobank. These are members of the NHLS Academic Affairs Research and Quality Assurance (AARQA), NHLS Biospecimen and Data Access Ethics Committee (BDAEC) committee, the Director of the NIOH, and representatives from existing biobanks within the NHLS [32]. The advisory committee consists of ten members and the strategic and organisational management of the NHLS 
Biobank is held accountable by this committee. Meetings are convened four times a year by the committee, and ad hoc meetings are convened should there be urgent matters to be addressed.

\section{External review}

The QMS of the NHLS Biobank and the effectiveness thereof is annually assessed through an external audit that is conducted by an international standards certification company in SA. The NHLS Biobank is certified for ISO 9001:2015 based on its QMS, traceability, and for adopting the highest level of best practice and standards. A prerequisite for undertaking research projects is ethics clearance for the study $[38,39]$. Ethics clearance is sought from Human Research Ethics Committee (HREC) in university institutions. Projects can only be initiated once a clearance certificate is issued.

\section{Internal procedures}

The NHLS Biobank has in place a model which ensures biobank processes are in line with international best practices and quality standards. These practices and standards are incorporated into internal procedures and documented in the form of SOPs, quality manuals, material transfer agreements and participant consent documents [32, 33]. A total of 21 internal Biobank SOPs have been established to maintain standardized and reproducible protocols relating to the pre-acquisition phase where consent is administered, to sample handling during its lifecycle in the biobank. Some of these SOPs are outlined in Table 2.

\section{Partnerships}

The NHLS Biobank is a member of different international societies and networks concerned with spreading excellence in all biobanking activities and cohort building. The NHLS Biobank participates through these professional organizations in creating standards and processes for biobanks globally [31]. In Addition to being an IBSER member, the NHLS Biobank is a member of European, Middle Eastern and African Society for Biobanking (ESBB) and Biobank and Cohort Building Network (BCNet). These partnerships enable the NHLS Biobank to keep abreast with new developments and adopt robust best practices. The NHLS Biobank is also in partnership with various government departments as well as national and international scientific communities to acquire the strategic and organisational management acumen for the national resource [32].

\section{Section B: Facilities}

\section{Temperature}

Ambient temperature is critical to the optimal operation of storage equipment and subsequently the integrity of samples. The NHLS Biobank currently has three freezer rooms with multiple freezer and fridge storage units, namely high-quality and energy efficient ultra-low temperature freezers $\left(-80^{\circ} \mathrm{C},-40^{\circ} \mathrm{C}\right.$, and $-20^{\circ} \mathrm{C}$ units), liquid nitrogen $\left(\mathrm{LN}_{2}\right.$ tank), fridges and ambient temperature storage rooms. As the storage equipment operate, the heat generated from the condenser fins is effectively dissipated by mechanical ventilation in the form of air conditioning systems. The temperature of storage equipment and the environment in which they operate under are fitted with temperature probes that are linked to an automated temperature logging system. The system has an in-built mobile phone Short Message Service (SMS) notification feature which notifies the manager of deviations from the set temperature ranges. Through this system corrective actions are initiated timeously and therefore equipment repair costs are kept at a minimum.

\section{Air flow, circulation and humidity}

Freezers, fridges and other equipment in the biobank are placed away from direct heat with space between them to allow air from the conditioning system to flow. Freezers and fridges are positioned at about an arm's length from the wall in order to increase air circulation. The room where the $\mathrm{LN}_{2}$ tank is placed is fitted with an oxygen monitoring system which provides both an audible and visible alarm to notify staff should the oxygen levels drop below optimum levels. Portable oxygen monitors (clipped onto lab coats) are also available to be used by staff when storing or retrieving samples from the tank. Personnel monitoring, through the buddy system is employed when the tank is operated.

An extraction fume hood system is installed for the processing of samples fixed in toxic solutions such as formalin and xylene. This system extracts toxic gases from the sample preparatory room which gets vented to the outside of the building. Another duct supplies fresh filtered air back into the room. Ventilation of the different sections of the biobank, including offices is controlled in such a way that it is adequate and conducive for human resources, infrastructural resources and repository operations. Samples requiring room temperature storage, such as histology and cytology samples (slides and block samples), humidity is prevented by air conditioning systems. 


\section{General lighting}

There is efficient artificial lighting in the facility adequate for the safe operation of the repository. The facility is fitted with light-emitting diode (LED) ceiling lights. The design of the facilities enables ambient natural light from the sun to penetrate through the windows. Natural light entering the room can be controlled by adjusting the fitted blinds, especially in office spaces where computers are operated where intense lighting can cause discomfort to staff. The walls of the facility are white in colour, which also enhances visibility and enables a safe working environment. In the event of power outages, the lights are powered by an automated generator.

\section{Flooring}

The NHLS Biobank is strategically located on the ground floor of the NIOH building. Majority of the storage equipment in the biobank are large in size and heavy and the ground floor is efficient in terms of accessibility and the safe movement of equipment should the need arise. The facility has durable, non-absorbent, low-maintenance flooring for the repository operations; vinyl laminate flooring (freezer rooms, walk-in freezers, sample processing rooms and offices) and ceramic tiles (kitchen). The flooring is permissible to traction, enabling the movement of new and old equipment possible across the floor area of the facility.

\section{Back-up power}

A $20 \mathrm{~L}$ automated generator provides continued power supply to the facility and all its equipment in the event of a power outage. In recent years, load shedding, which is a planned interruption of power supply when the demand for electricity exceeds the available supply was introduced in SA by the government through Eskom, an SA electricity public utility [40]. The automated generator has provided continued electricity supply in all power outages. This is especially critical for specimen storage equipment to ensure that an optimal temperature for sample integrity is maintained as appropriate. Furthermore, the storage equipment is also connected to carbon dioxide $\left(\mathrm{CO}_{2}\right)$ cylinders as an additional back-up should the generator fail to engage during a power outage. Service and maintenance of the generator is of importance and this is planned for and carried out by the maintenance department of the institution.

\section{Security and access}

Safeguarding HBMs is a crucial function of the NHLS Biobank. Investing in infrastructure that provides the highest level of security and protection of resources through engineering and administrative controls is a key priority for the NHLS biobank. The facility is equipped with restricted electronic access control system and CCTV cameras to ensure biomaterials and data privacy and protection. The system prevents unauthorised access to the facility which is only limited to biobank staff. Freezers are locked at all times, and records of access to samples and data are kept. Access to the Laboratory Information Management System (LIMS) is password protected and activities in the system are traceable to the individual performing a task to uphold confidentiality and the interests of research participants. There are security guards available on a daily basis to safeguard the premises and report alarms as well as prevent physical intrusion of unauthorised individuals. Visitors to the biobank sign a visitors' register followed by induction and are always accompanied by a staff member. Visitors are given a tag which should be visible on them and identifies them as visitors. Records of the visitors' register is filed for record keeping.

\section{Fire prevention system}

The biobank has an appointed fire warden who has undergone the necessarily training and deemed competent to safeguard the facility from preventative fires. Conducting fire safety checks and ensuring that all fire prevention equipment is well maintained are the responsibilities of the fire warden.

The facility is fitted with smoke detectors equipped with audible and visible alarms, and emergency break glass units to disconnect the electronic access control security system in the event of fire to allow staff to swiftly evacuate the building. Fire extinguishers $\left(\mathrm{CO}_{2}\right.$, and dry chemical powder) are located throughout the sections of the facility and a fire blanket is placed in the kitchen. A fire hydrant for class A fires is also installed in the facility. The fire prevention system for the facility is centred around preventative maintenance and repairs of all electronic systems, equipment, electric works and cables as well as the proper storage of flammable liquids.

\section{Emergency preparedness}

The NHLS Biobank has a disaster management plan adopted from the NHLS disaster management guidelines 
[32, 41, 42]. The disaster management guidelines of the NHLS, adopted from the Disaster Management Act 57 of 2002, makes provision for the handling of disasters that are natural or human-caused that may threaten or cause death, injury or disease, damage to property, infrastructure or the environment [32, 41]. The NHLS guideline was modified to relate specifically to disasters likely to affect the NHLS repository operations [42]. The effectiveness of the emergency evacuation plan and procedure is verified through planned and unplanned drills where real life scenarios are simulated.

\section{Pest and contamination control}

The Facilities Department of the NIOH is responsible to ensure the building is free of pests by monitoring and requesting pest control treatment should the building be infested with pests. The NHLS Biobank has an appointed Health and Safety Representative (HSR) trained and deemed competent to conduct checks for health and safety hazards.

\section{Relocation of repository}

Provision has been made with a laboratory of the NHLS and an academic institution for the relocation of biological resources to their facilities. These facilities have reserved storage rooms that are accommodative for the needs of the NHLS Biobank in the event of disaster. The process of establishing other biobanks of the National Biobank is underway in other provinces, which will enable the movement of biological resources from one facility to another if disaster strikes.

\section{Section C: Storage and processing equipment}

Biobank equipment indicated in Section A are traceable through an Equipment Inventory Log where the equipment is assigned a unique asset number. Elaborate procedures to follow for equipment maintenance, equipment downtown, and unit decontamination is provided in SOPs. A yearly calibration and preventative maintenance/service plan is scheduled and conducted for all equipment by service providers accredited by South African National Accreditation System (SANAS). Storage equipment in the NHLS Biobank is accommodative for a variety of samples requiring short-term and long-term storage. The requirements for service are discussed during the initial engagement with potential clients [23].

\section{Section D: Quality management}

ISBER Best Practices for Biorepositories was instrumental in the development of the infrastructure and QMS for the NHLS Biobank. In April 2019, the NHLS Biobank met the QMS requirements for ISO 9001:2015 and was certified after it was assessed for compliance in terms of providing products and services that consistently meet customer requirements and evidence of continual improvements in the QMS. Having met the requirements for ISO 9001:2015 certification, which satisfies the quality assurance (QA) aspect of the QMS, provision for the implementation of ISO 20387, overarching the guiding principle of suitability specific to biobanking is underway for the NHLS Biobank. Accreditation with ISO 20387:2018 together with the quality control (QC) assays implemented for the biobanking processes will confirm the reliability of the biological resources in the NHLS Biobank. This will propel the NHLS Biobank towards integration and harmonization, thereby enabling the sharing of high-quality and well-annotated biological resources that will contribute to modern biomedical research on an international scale [8].

\section{Section E: Method validation and quality control considerations}

Methods are adopted or developed and validated to execute the different phases of the NHLS biobanking processes while preserving the quality and relevance of the samples in the long-term [43, 44]. Different parameters of analysis are used for validation in order to effectively detect defective outputs of methods employed to carry out the biobanking processes. QC measures are conducted using certified reference material that establishes tractability of the accurate value and establishes the stated level of confidence. QC protocols employed depend on the sample type and utility of the samples. Section K provides more information specific to the collection and storage phases of the biobank process with relevance to method validation and QC considerations.

\section{Section F: Safety}

A safety plan which ensures the safe operation of the biobank for the protection of staff and visitors was developed through a comprehensive risk assessment which involved the overall process of accurate identification, analysis and evaluation of risks inherent to the operational activities and layout of the biobank. Hazards to the health and safety of employees and visitors are identified for the NHLS 
Biobank and these are categorised as biological, chemical, physical and psycho-social hazards. Examples of the categorised hazards are shown in Table 3.

The highest level of control is implemented where reasonably practicable, to completely eliminate exposure of staff and visitors to the identified hazards in the longterm or to minimize exposure to the hazard and this was achievable by applying the principles of hierarchy of hazard control [45]. The NHLS Biobank adheres to the occupational health and safety management system of the NHLS which is governed by the Occupational Health and Safety Act (OHS Act 85 of 1993) [46], an SA regulation governing the safety and health of employees and persons that might be affected by the work being conducted.

Biobank staff are supplied with adequate personal protective equipment (PPE). For every task or procedure carried out, the required PPE and equipment to ensure safety of the staff is outlined in SOPs as well as in the risk assessment document. Health and safety related training and information is available on the NHLS intranet for all staff. Due to the variety of samples handled in the Biobank, staff are vaccinated against blood borne pathogens such as hepatitis $\mathrm{B}$, and baseline X-ray checks are done for tuberculosis, followed by screening which is self-administered quarterly. Covid-19 control measures for infection prevention in the workplace which includes daily screening of biobank staff for Covid-19 symptoms is also administered.

\section{Section G: Training}

The NHLS Biobank staff are comprised of laboratory clerks and medical scientists who report to the manager. Staff are responsible for the daily operations of the Biobank and are assigned responsibilities based on their job description and competencies. Training is an integral part of competence determination. Staff have the necessary qualifications, training, and experience to effectively and safely

Table 3: Hazards to the health and safety of employees and visitors.

\begin{tabular}{ll}
\hline $\begin{array}{l}\text { Hazard } \\
\text { classification }\end{array}$ & Examples of hazards \\
\hline Biological & $\begin{array}{l}\text { Blood borne infectious agents in body fluids and } \\
\text { tissues (hepatitis B, HIV, TB etc.) } \\
\text { Flammable and toxic chemicals and gases } \\
\text { (alcohol, formalin, xylene, LN }\end{array}$ \\
Physical & $\begin{array}{l}\text { Frost bites from ultra-low freezers, hypothermia } \\
\text { in walk-in freezer, asphyxiation from liquid } \\
\text { nitrogen gas }\end{array}$ \\
& Dealing with dissatisfied clients or stakeholders \\
\hline
\end{tabular}

operate the biobank. Competencies and qualifications of staff are filled in their respective personnel files for record keeping. Knowledge and practice is kept up to date by reviewing staff training needs annually or as changes to the system are effected. Determination of personnel competence is identified through task appropriate academic qualifications and training, and this is structured as part of a broader quality system within the biobank operation [32]. Key performance indicators (KPIs) are set annually and capabilities and needs of staff are detectable during staff appraisals and there are procedures in place that promote staff progression. Personnel complement of the biobank is indicated in Table 4 with the respective KPI aspects. Over and above the provision of storage services, the NHLS Biobank conducts teaching and training of medical students in universities on biobanking, training of other interested parties on starting biobank operations nationally and internationally. Furthermore, the NHLS Biobank provides sample reception services for departments within the NIOH and NHLS Central Laboratory Support Services (CLSS).

\section{Section H: Cost management}

The NHLS Biobank is a non-profit generating entity operating on a cost recovery business model. Resources required for optimum functioning of the biobank were forecasted during the design and development planning of the NHLS Biobank which continuously informs annual budgetary allocations and sustainability of the model by Management and key stakeholders. The NHLS Biobank receives its annual budget from national treasury and these

Table 4: Biobank personnel and their respective KPIs.

\begin{tabular}{ll}
\hline Personnel & KPI aspects \\
\hline Clerks $(n=4)$ & (1) Collection of specimen \\
& (2) Registration of specimen \\
& (3) Storage of specimen \\
& (4) Dispatch of specimen \\
Medical scientists ( $n=2)$ & (1) Research and development (R\&D) \\
& (2) Scientific writing \\
& (3) Research funding \\
& (4) Teaching and training \\
Biobank manager $(n=1)$ & (5) Quality management system (QMS) \\
& (2) Biobank daily operations \\
& (3) Biobank budget management \\
(4) Biobank quality management & (5) Biobank health and safety management
\end{tabular}


funds are administered by the NHLS to its divisions. The annual budget of the NHLS from national treasury dictates the percentage allocated to the NHLS Biobank. The budget ranges between 5 and $10 \%$ of the overall budget allocated for operational activities by the NHLS. Over and above the allocated budget, the NHLS biobanking services generate revenue that is reinvested into the model as a cost recovery strategy.

The assessment of sources of costs, which is done for infrastructural and operational needs of the NHLS Biobank, is employed to control costs. Sustainability heavily depends on how well the activities concerned with the biobank are translated into cost information that prompts decision making favourable to sustain the business model in the long-term by maximizing outputs with the least costs, a method called cost minimisation [47].

Biobank daily operations are costly and management has to choose from economic efficiency to technical efficiency. The biobank input costs involve labour, equipment service and calibration, electricity, sample transport cars and fuel, liquid nitrogen, training and education, certification and membership costs.

Factors such as inflation affects the budget of the biobank as this directly result in increased cost recovery charges. Cost is managed by monthly stock count and checking the utilisation of inputs. The amount of labour needed in the biobank is constantly scrutinised against the method used to collect and store samples and the associated data. Energy efficient processing and storage equipment are employed to keep operating costs to a minimum.

\section{Section I: Repository management information system}

The NHLS Biobank is equipped with an electronic inventory system for the management and accounting of biological samples and their data. The physical storage of samples and their associated data is configured and traceable on the password protected system, to ensure biomaterials and data privacy and protection $[32,48]$. The biobank LIMS not only enables tracking of sample usage, movement within the repository, retrieval and dissemination to clients, and sample return from clients, it also permits search and query abilities for all data stored. Critical to the synergy between the physical and electronic storage of samples is the sample unique identifier and the accurate labelling of samples and capturing of data. Samples in the NHLS Biobank are assigned unique identification numbers and labelled as such using the barcode system readable to humans and computers. The usage of a barcode scanner for data input is employed to prevent transcription errors. Unique identification numbers are employed to de-identity personal information traceable to the donor.

The extent to which staff can access and use the system is task dependent and user restrictions are configured by the manager for the purpose of data protection and upholding confidentiality for those that have donated the samples. Activities of staff on the system is traceable to an individual and this is ensured by staff having personal login credentials used when they access the system. An audit trail and periodic repository inventory is conducted which can either be regular or random to ensure the effectiveness of the system as intended for biobanking processes. The system is programmed to log off users after a specified time of inactivity while logged on to the system. Data in the system is frequently backed-up to protect against data loss. The back-end of the system is maintained by the Information Technology Department.

\section{Section J: Packaging and shipping}

Within the context of a biobank, retention of sample integrity and stability as much as possible is crucial. This ensures quality of the stored samples, consequently reliability of the data generated from processing of samples. Provision for procedures applicable to road and air transporting of samples is necessary. The Biobank takes into account considerations for infectious and diagnostic substances, classified by International Air Transport Association (IATA) as dangerous goods [49]. Key factors considered in ensuring adequate sample preservation include; (1) efficient logistics system, (2) availability of constant power (back-up power in cases of power failure), (3) constant availability of frozen ice packs or dry ice [30, 50,51]. The Biobank is compliant with IATA guidelines as demonstrated by personnel that have been trained and certified accordingly. This ensures control and mitigation of potential risk factors that may influence sample quality including; (1) limited space for biobank sample transport when delivery is combined with other laboratory sample deliveries and (2) turn Around Times (TATs) for sample shipping. Availability of at least one vehicle dedicated to biobank sample shipping is highly recommended. The biobank utilizes its own transport system with access to vehicle service records as a means of assessing transport efficiency to avoid breakdowns which might compromise sample quality and integrity. Sample cold chain during shipping is maintained using ice packs or dry ice. Digital thermometers are used for monitoring of transit temperatures according to manufacturer's instructions translated 
into SOPs. Digital thermometers are typically used with software that enables retrieval of transit temperature recordings (pre- and post-delivery readings). Transit temperature readings are assessed for any temperature deviations and all records kept.

\section{Section K: Specimen collection, processing, receiving, and retrieval}

The process of biobanking, which includes sample and data collection, processing, receiving and retrieval is documented in SOPs. There are quality checks for each of the processes listed above to ensure that the process and the end product is fit for purpose. These checks include competency of staff involved, effectiveness of equipment and method employed, sample integrity and viability through transit temperature monitoring and recording, as well as $100 \%$ error checks for transcription errors for sample registration. For instance, during collection, in-transit temperature of samples is monitored to ensure that cold chain is maintained while samples are in-transit to and from the NHLS Biobank, especially for temperature sensitive samples. This is achieved by using calibrated electronic temperature logging devices against a reference standard from a SANAS accredited facility. The temperature of samples during transit to the biobank is documented together with other pre-analytical variables inherent to the collection, processing and storage phases of the biobank lifecycle. Knowledge of pre-analytical variables inherent to the biobanking processes is fundamental for downstream applications and accurate interpretation of research results derived from the samples [23, 43, 44].

Conditions of the Biobank storage equipment are assessed through inter-laboratory storage of QC samples and subsequent testing of these samples to determine reproducibility of results run in parallel to those stored at another laboratory over a period of time using baseline results as reference (Figure 2A-E). The findings of the QC checks are documented and corrective actions are effected where there are discrepancies. These processes are conducted by trained and competent staff and each process has a workflow authorised by the manager. As indicated in Section L below, there are qualifying criteria to be met before initiating collections and the exchange of samples between involved parties ensues. Liquid samples are stored in aliquots to avoid multiple freeze-thaw cycles of the entire quantity of a given sample at a given time, and these are stored in different storage units. Multiple samples from individual patients are frequently collected for redundancy.

The unique identifier for samples ensures efficient traceability for retrieval purposes from the inventory

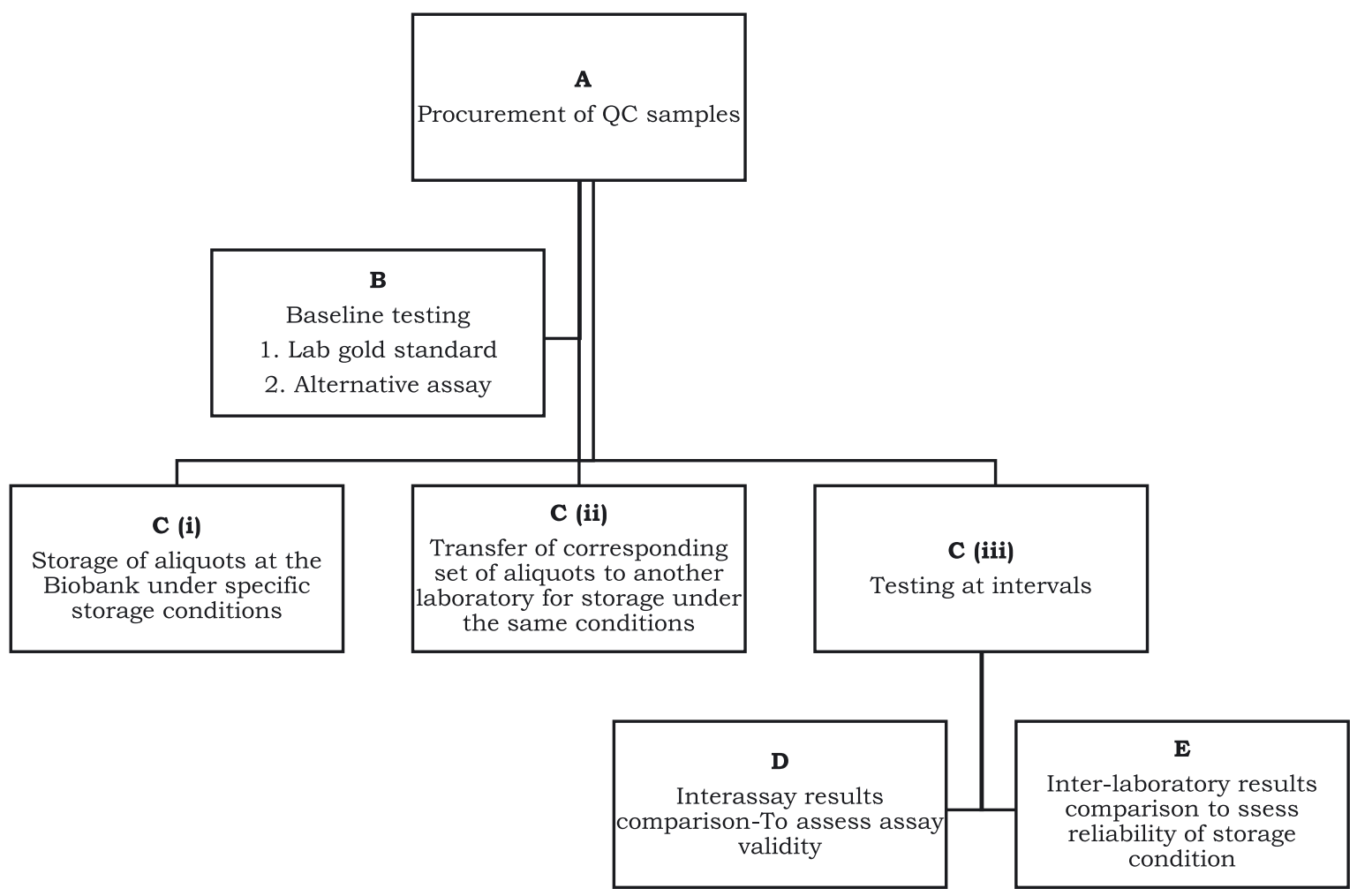

Figure 2: Process flowchart for optimising storage conditions for biospecimens at the NHLS Biobank. 
system. As with the collection stage, the retrieval of samples is also documented and factors such as time, temperature, person involved in the retrieval process and the reason(s) for retrieval are documented and subjected to QC. These processes must also be traceability on the inventory system. Internal or external factors that pose as a risk to the efficient operation of the biobank activities is documented on a risk register and mitigations strategies to lower the risk or eliminate it are effected.

\section{Section L: Legal and ethical issues for biospecimens}

There are no biobank specific regulations and legislation in SA, therefore, a number of national regulatory instruments and legislation as well as international guidelines and declarations govern the NHLS Biobank processes and research [38, 39, 52]. MTAs are signed by all parties prior any transfer of material (specimens and associated data). In the MTA, rights and responsibilities of both the Biobank (provider of service) and the transferring institution (receiver of service or custodian) are clearly stipulated and defined [52-54]. The MTA also protects the rights of people from whom the materials have been collected in line with principles of ethics as well as the law and regulations [52-54]. Some of the elements of the MTA that ensure sound ethical conduct and adherence to the law include a requirement for HREC review prior material transfer by the custodian to the Biobank, custodianship and benefit sharing as well as a responsibility for privacy and confidentiality by the Biobank [52-54]. This ensures respect for participant autonomy while public (community) engagement activities ensure beneficence for SA communities by distributing the relevant biobank and biobank research information which in turn strengthens participant autonomy, i.e. providing full information necessary for the consent process. The transfer of materials back to the custodian is only released upon permission by the custodian to prevent inappropriate sharing and use.

\section{Section M: Specimen access, utilization, and disposition}

Clients of the NHLS Biobank are custodians of the samples and their associated data currently in storage, third party access to these collections is protected as per ethical and legal requirements [32]. Samples can be collected and distributed to NHLS researchers, private researchers and industry on the conditions of joint collaboration projects using samples that have been collected using broad consent [55]. Requests to biospecimen, data and resources access for research from the NHLS is facilitated by AARQA and the process workflow is illustrated in Figure 3 [55]. All applications are registered and submitted on the Academic Affairs and Research Management System (AARMS) of the NHLS and the applications are processed by the Academic Affairs Research (AAR) Office which forms part of AARQA [55]. Access criteria is facilitated as a function of scientific value and ethical soundness of the proposed project(s). Under no circumstances are the specimens and associated data used by Biobank personnel or third parties unless otherwise agreed upon and in collaboration with the custodians. To date, $20 \%$ of the total number of samples that were in storage (plasma, serum, urine, histology slides and block samples) have been retrieved for research and pathology referral purposes by custodians.

Disposal of specimens at the Biobank is regulated by the National Waste Act No. 59 of 2008 [56], as well as Occupational Health and Safety (OHS) rules. All of the specimens to be disposed of are considered biohazardous and the appropriate procedure for discarding biohazardous biomaterial is followed. Physical disposal of specimens is preceded by destruction on LIMS.

The flowchart is adopted from the flowchart for the application and submission process outlined by AAR to access biospecimens and/or data from the NHLS for research purposes [55].

\section{Future considerations for the NHLS Biobank}

The NHLS Biobank has made significant advancements which has seen it develop into an entity with infrastructure and operational activities that support its short-term and long-term objectives set out in the business plan. The successful establishment and operation of the NHLS Biobank is owed to its stakeholders. This necessitates the continued practice of nurturing mutually beneficial partnerships with stakeholders while upholding the interests of donors in order for NHLS Biobank to remain a functional support infrastructure for valuable research in the longterm.

To ensure sustainability, probing the needs of academic researchers and industry will be crucial in order for the derived services and products to be aligned to and bridge gaps relating to access to high quality samples and wellannotated data for medical research and discovery. Supporting priority health programs of the National Department 


\section{Registration}

AARMS System (https://aarms.nhls.ac.za) -----> Application registration Researcher (Principal Investor and/or Co-Researcher)

\section{Submission of Application}

AARMS System (login credential from registration). Research application submission with suporting documents (ethics approval \& other relevant documents)

\section{Aknowledgment of Receipt}

Project application assigned a number. Aknowledgment of receipt of application by AAR (email notification to applicant)

\section{Review of Application Content \\ Internal review (feasibility, content, ethical soundness, \& scientific meritetc.) \\ Complex requests --> BDAEC for further review \& external review}

\section{Outcome Notification}

Outcome of application communicated via email; Approval, Rejection or Ammendment letters

\section{Data and/or Biospecimen Request}

Request data and/or biospecimen preparation

\section{Release of Data and/or Biospecimen}

Approved data and/or biospecimen released
Figure 3: Flowchart for the application process for access to biospecimens, data and resources of the NHLS for research purposes. of Health by presenting viable solutions to academic researchers and industry may result in long-term investments from these parties. These partnerships may yield to access to mixed-funding schemes which may enable the NHLS Biobank to hedge against financial implications inherent to public biobanks.

Another function of sustainability is an efficient costaccounting tool, which will need to be reviewed and modified as the NHLS Biobank operates and expands in scope and size to maximise its capacity of making accessibility to large numbers of diverse samples and associated data possible to the research community.

Cutting down costs, identifying and adopting costrecovery strategies will be paramount in the long-term. Provision for employing cost-effective storage solutions such as the freeze drying of samples and storage at ambient temperature without compromising sample integrity is underway. This will reduce costs associated with procuring and maintaining ultra-low temperature freezer storage units. Automation of biobanking processes such as sample processing, storage and retrieval are hoped to keep costs associated with human resources at a minimum while maximising high quality outputs. Provision for virtual collections of pathology samples is also underway at the NHLS Biobank to enable efficient accessibility and retrieval of digitised catalogues of specimens on physical slides for diagnostic and research purposes.

The NHLS Biobank endeavours to reach a state of interoperability with other biobanks and this is achievable through networking and uniformity of biobanking processes. Uniformity of processes will ensure that the quality of samples is the same irrespective of where they are collected. Provision for the implementation of ISO 20378:2018 is underway which will demonstrate technical competence of the NHLS Biobank to provide high quality samples and well-annotated data suitable for downstream applications. This milestone will solidify total QMS of the biobank and present an opportunity for the national treasure to be integrated and harmonised with other biobanks on an international scale. 
The ethical framework of biobanking is contentious with new arising issues as the scope and size of biobanks expand. The NHLS Biobank will need to keep abreast with all developments pertaining to the ethics and governance of biobanking to ensure that acquisition and distribution of biological resources is conducted under a well-defined ethical and legal framework.

The NHLS Biobank must continuously broaden its reach by disseminating information on the role, activities and interests of the biobank to the public and health professionals. It has become evident in literature that the extent of biobanking knowledge amongst health professionals is not well investigated, albeit the effective collection of samples from donors requires the involvement of health professionals from the ward to the laboratory before reaching the biobank [28]. The involvement of health professionals can also ensure an effective collection system of samples through teaching and training to control and evade pre-analytical variables that are inevitable during sample collection. This will introduce quality control measures from the collection site and throughout the lifecycle of samples and data in the biobank, fostering reliability and accuracy of biological material for valuable research.

Acknowledgments: We thank the National Institute for Occupational Health, a division of the National Health Laboratory Service, and the NHLS Biobank and Quality Assurance personnel.

Research funding: The National Health Laboratory Service funded the publication.

Author contribution: All the authors have accepted responsibility for the entire content of this submitted manuscript and approved submission.

Conflict of interest statement: The authors declare no conflicts of interest regarding this article.

Informed consent: Not applicable.

Ethical approval: Not applicable.

Data availability: Information supporting the findings presented in the article are available from the corresponding author, M.S., on request.

Disclaimer: The views expressed in the submitted article are those of the authors and not an official position of the institution.

\section{References}

1. Organisation for Economic Co-operation and Development. Creation and governance of human genetic research databases. Available at: https://stats.oecd.org/glossary/detail.asp?
ID=7220\#: :text=0ECD\%20Statistics,)\%20or\%20\%E2\%80\% 9Cpopulation\%20database\%E2\%80\%9D [Accessed 12 Jan 2021].

2. Liaño F, Torres AM. Biobanks: a new tool for clinical research. Nefrologia 2009;29:193-5.

3. Biobanking and Biomolecular Resources Research Infrastructure (BBMRI). Biobanks and the public: governing biomedical research resources in Europe. Available from: https://www. bbmri-eric.eu/wp-content/uploads/BBMRI-Biobanks-and-thePublic.pdf [Accessed 12 Jan 2021].

4. Hewitt R, Watson P. Defining biobank. Biopreserv Biobanking 2013;11:309-15.

5. Kinkorová J. Biobanks in the era of personalized medicine: objectives, challenges, and innovation. EPMA J 2016;7:4.

6. Coppola L, Cianflone A, Grimaldi AM, Incoronato M, Bevilacqua P, Messina F, et al. Biobanking in health care: evolution and future directions. J Transl Med 2019;17:172.

7. Malsagova K, Kopylov A, Stepanov A, Butkova T, Sinitsyna A, Izotov A, et al. Biobanks-a platform for scientific and biomedical research. Diagnostics 2020;10:485.

8. Meinung B, Martin D, Zimmermann W. Standardization in biobanking - between cooperation and competition. J Lab Med 2019;43:317-28.

9. Moodley K, Singh S. "It's all about trust": reflections of researchers on the complexity and controversy surrounding biobanking in South Africa. BMC Med Ethics 2016;17:57.

10. Betsou F, Luzergues A, Carter A, Geary P, Riegman P, Clark B, et al. Towards norms for accreditation of biobanks for human health and medical research: compilation of existing guidelines into an ISO certification/accreditation norm-compatible format. Qual Assur J 2007;11:219-92.

11. Hewitt RE. Biobanking: the foundation of personalized medicine. Curr Opin Oncol 2011;23:112-9.

12. Budimir D, Polasek O, Marusić A, Kolcić I, Zemunik T, Boraska V, et al. Ethical aspects of human biobanks: a systematic review. Croat Med J 2011;52:262-79.

13. Prictor $\mathrm{M}$, Teare $\mathrm{H}$, Kaye J. Equitable participation in biobanks: the risks and benefits of a "dynamic consent" approach. Front Public Health 2018;6:253.

14. Moore HM, Kelly A, Jewell SD, McShane LM, Clark DP, Greenspan $\mathrm{R}$, et al. Biospecimen reporting for improved study quality. Biopreserv Biobanking 2011;9:57-70.

15. De Souza YG, Greenspan JS. Biobanking past, present and future: responsibilities and benefits. AIDS 2013;27:303-12.

16. Zawati MH, Knoppers B, Thorogood A. Population biobanking and international collaboration. Pathobiology 2014;81:276-85.

17. Asslaber M, Zatloukal K. Biobanks: transnational, European and global networks. Briefings Funct Genomics Proteomics 2007;6: 193-201.

18. Grizzle WE, Gunter EW, Sexton KC, Bell WC. Quality management of biorepositories. Biopreserv Biobanking 2015;13:183-94.

19. BiobankCohort Building Network. Partners - organizations and networks. Available from: https://bcnet.iarc.fr/about/partners. php [Accessed 18 Jan 2021].

20. Campbell LD, Astrin JJ, DeSouza Y, Giri J, Patel AA, Rawley-Payne $M$, et al. The 2018 revision of the ISBER best practices: summary of changes and the editorial team's development process. Biopreserv Biobanking 2018;16:3-6.

21. Vaught J, Lockhart NC. The evolution of biobanking best practices. Clin Chim Acta 2012;413:1569-75. 
22. Simeon-Dubach D, Zeisberger SM, Hoerstrup SP. Quality assurance in biobanking for pre-clinical research. Transfus Med Hemotherapy 2016;43:353-7.

23. Valdivieso-Gómez V, Garrancho-Pérez J, Aroca-Siendones I, Aguilar-Quesada R. Harmonized and quality sample handling in biobank-supported multicenter prospective studies. In: Erhabor 0, Munshi A, editors. Human blood group systems and haemoglobinopathies. IntechOpen; 2020. Available from: https://www.intechopen.com/chapters/70920 [Accessed 12 Jan 2021].

24. Organisation for Economic Co-operation and Development. OECD best practice guidelines for biological resource centers - general best practice guidelines for all BRCs. Available from: https:// www.oecd.org/officialdocuments/publicdisplaydocumentpdf/? cote $=\mathrm{dsti} / \mathrm{stp} / \mathrm{bio}(2007) 9 /$ final\&doclanguage $=$ en $[$ Accessed 18 Jan 2021].

25. Organisation for Economic Co-operation and Development. OECD guidelines on human biobanks and genetic research databases. Available from: http://www.oecd.org/sti/emerging-tech/ 44054609.pdf [Accessed 19 Jan 2021].

26. International Organization for Standardization. ISO 9001: 2015(en) Quality Management Systems-Requirements. Available from: https://www.iso.org/obp/ui/\#iso:std:iso:9001:ed-5:v1:en [Accessed 19 Jan 2021].

27. International Organization for Standardization. ISO 20387:2018 Biotechnology-Biobanking-General Requirements for Biobanking. Available from: https://www.iso.org/standard/ 67888.html [Accessed 19 Jan 2021].

28. Kintossou AK, N'dri MK, Money M, Cissé S, Doumbia S, Soumahoro M-K, et al. Study of laboratory staff' knowledge of biobanking in Côte d'Ivoire. BMC Med Ethics 2020;21:88.

29. Mohammadi M. Biobanking in the developing world; Maximum specimens, minimum infrastructure. Basic Clin Cancer Res 2018; 9:1-3.

30. Abayomi A, Christoffels A, Grewal R, Karam LA, Rossouw C, Staunton $C$, et al. Challenges of biobanking in South Africa to facilitate indigenous research in an environment burdened with human immunodeficiency virus, tuberculosis, and emerging noncommunicable diseases. Biopreserv Biobanking 2013;11:347-54.

31. National Biobank of the National Health Laboratory Service. Biobank video profile. Available from: https://www. nationalbiobank.nhl.ac.za [Accessed 3 Feb 2021].

32. Maseme M. Biobank Quality Manual. BIOB0008, vol 7; 2020:1-41 p. (unpublished: internal standard operating procedure).

33. Maseme M. Governance for the NHLS Biobank. BIOB0019, vol 1; 2020:1-12 p. (unpublished: internal standard operating procedure).

34. Gille F, Vayena E, Blasimme A. Future-proofing biobanks' governance. Eur J Hum Genet 2020;28:989-96.

35. De Vries J, Munung SN, Matimba A, McCurdy S, Oukem-Boyer OOM, Stauton C, et al. Regulations of genomic and biobanking research in Africa: a content analysis of ethics guidelines, policies and procedures from 22 African countries. BMC Med Ethics 2017;18:8.

36. Andanda P, Govender S. Regulation of biobanks in South Africa. J Law Med Ethics 2015;43:787-800.

37. Dhai A, Mahomed S, Sanne I. Biobanks and human health research: balancing progress and protections. S Afr J Bioeth 2015; $8: 55$.
38. Government of South Africa. Regulations relating to research with human participants GNR719 GG 38000 of 19 September 2014. Available from: http://www.gpwonline.co.za/Gazettes/ Gazettes/38000_19-9_NationalRegulation.pdf [Accessed 2 Feb 2021].

39. Department of Health of South Africa. Ethics in Health Research: principles, processes and structures, 2nd ed. Pretoria: Department of Health; 2015. Available from: https://www.ru.ac. $\mathrm{za} /$ media/rhodesuniversity/content/ethics/documents/ nationalguidelines/DOH_(2015)_Ethics_in_health_research_ Principles,_processes_and_structures.pdf [Accessed 2 Feb 2021].

40. City of Johannesburg. What is load shedding. Available from: https://www.joburg.org.za/departments_/Pages/MOEs/city\% 20power/What-is-loadshedding.aspx\#: :text=Load\% 20shedding\%20plans\%20for\%20Joburg\&text=When $\% 20$ the $\%$ 20demand $\% 20$ for $\% 20$ electricity,electricity $\% 20$ between $\% 20$ all \%20Eskom\%20customers [Accessed 3 Feb 2021].

41. Working Group-Safety. Disaster management plan. GPS0036, vol 2; 2019:1-7 p. (unpublished: internal standard operating procedure).

42. Maseme M. National Health Laboratory Service Biobank disaster management plan. BIOB0016, vol 1; 2019:1-6 p. (unpublished: internal standard operating procedure).

43. Betsou F, Gunter E, Clements J, DeSouza Y, Goddard KA, Guadagni $F$, et al. Identification of evidence-based biospecimen qualitycontrol tools: a report of the international society for biological and environmental repositories (ISBER) bioscience working group. J Mol Diagn 2013;15:3-16.

44. Lehmann S, Guadagni F, Moore H, Ashton G, Barnes M, Benson E, et al. International society for biological and environmental repositories (ISBER) working group on biospecimen science. Standard preanalytical coding for biospecimens: review and implementation of the sample PREanalytical code (SPREC). Biopreserv Biobanking 2012;10:366-74.

45. National Institute for Occupational Safety and Health. Hierarchy of controls. Available from: https://www.cdc.gov/niosh/topics/ hierarchy/default.html [Accessed 3 Feb 2021].

46. Department of Labour. Occupational Health and Safety Act 85 of 1993. Available from: https://www.gov.za/documents/ occupational-health-and-safety-act\# [Accessed 3 Feb 2021].

47. Gonzalez-Sanchez MB, Lopez-Valeiras E, García-Montero AC. Implementation of a cost-accounting model in a biobank: practical implications. Pathobiology 2014;81:286-97.

48. Maseme M. Pathogen asset control system procedure for the NHLS Biobank. BIOB0020, vol 1; 2021:1-15 p. (unpublished: internal standard operating procedure).

49. International Air Transport Association. Dangerous Goods Regulations. Available from: https://www.iata.org/en/ programs/cargo/dgr/download [Accessed 3 Feb 2021].

50. Dickinson B. Specimen Collection. Available from: http://www. bd.com/vacutainer/pdfs/techtalk/TechTalk_November2011.pdf [Accessed 3 Feb 2021].

51. Maseme M. Collection, transporting, receiving and storage of specimens for biobanking purposes. BIOB0004, vol 4; 2020:1-12 p. (unpublished: internal standard operating procedure).

52. Government of South Africa. Material transfer agreement for human biological materials government notice 719 , government gazette 41781 of 20 July 2018 . Available from: https://www.gov. 
za/sites/default/files/gcis_document/201808/41781gon719.pf [Accessed 2 Feb 2021].

53. Thaldar DW, Botes M, Nienaber A. South Africa's new standard material transfer agreement: proposals for improvement and pointers for implementation. BMC Med Ethics 2020;21:85.

54. Bubela T, Guebert J, Mishra A. Use and misuse of material transfer agreements: lessons in proportionality from research, repositories, and litigation. PLoS Biol 2015;13:e1002060.
55. Mayne E, Malope-Kgokong B. Research material and data access policy. POLR0006; 2020, vol. 1:1-14 pp. (unpublished: internal standard operating procedure).

56. University of Pretoria and Southern African Legal Information Institute. National Environmental Management: Waste Act 59 of 2008. Available from: https://cer.org.za/wp-content/uploads/ 2010/03/59-OF-2008-NATIONAL-ENVIRONMENTAL-MANAGEMENTWASTE-ACT_2-Sep-2014-to-date.pdf [Accessed 5 Feb 2021]. 